\section{Inorganic chiral 3-D photonic crystals with bicontinuous gyroid structure replicated from butterfly wing scales $\dagger$}

\author{
Christian Mille, ${ }^{a b}$ Eric C. Tyrode ${ }^{c}$ and Robert W. Corkery ${ }^{* a b}$ \\ Received 21st March 2011, Accepted 12th July 2011 \\ DOI: $10.1039 / \mathrm{c} 1 \mathrm{cc} 11637 \mathrm{a}$
}

Three dimensional silica photonic crystals with the gyroid minimal surface structure have been synthesized. The butterfly Callophrys rubi was used as a biotemplate. This material represents a significant addition to the small family of synthetic bicontinuous photonic crystals.

Photonic crystals are ordered materials used to manipulate light by preventing the propagation of certain wavelengths. Their photonic properties vary mainly as a function of dielectric properties, topology, periodicity and symmetry of the crystal structure. ${ }^{1}$ To date most photonic crystals are two-dimensional and manufactured with methods borrowed from the semiconductor industry. ${ }^{2}$ Recent calculations have shown that triply-periodic minimal surfaces (TPMS), like the cubic gyroid, ${ }^{3}$ are good candidates for making photonic crystals with full band gaps than can potentially be tuned in the whole visible spectral range by varying the lattice constant and refraction index contrast. ${ }^{4}$ Nonetheless, the manufacturing of such 3-D crystals have so far been difficult. Top-down approaches including photolithography and etching techniques are cumbersome when used for 3-D structures. Bottom-up approaches using self assembled colloids or block copolymers often yield crystal structures with stacking faults or are limited to length scales less than the wavelength of visible light. ${ }^{5}$ Using naturally occurring photonic crystals as biotemplates represent an obvious way forward. Bicontinuous cubic structures are not uncommon in nature. Larsson et al. ${ }^{6,7}$ pioneered the study of bicontinuous cubic structures in cellular membranes, finding numerous examples in species from all kingdoms, for example, in various organelles such as chloroplasts ${ }^{8}$ mitochondria ${ }^{9}$ and endoplasmatic reticula (ER). ${ }^{10}$ However, they are usually not suitable as templates for optical photonic crystals because their lattice constants are too small. The ordered biopolymeric structures used by many animals to display colours are better candidates. ${ }^{11}$ Examples of these biological 'structural colours'

${ }^{a}$ YKI, Institute for Surface Chemistry, SE-114 86, Stockholm, Sweden.E-mail: robert.corkery@yki.se; Tel: +4610 5166000

${ }^{b}$ Berzelii Centre EXSELENT on Porous Materials, Stockholm University, Stockholm, Sweden

${ }^{c}$ Surface and Corrosion Science, Department of Chemistry, Royal Institute of Technology, SE-100 44, Stockholm, Sweden

$\dagger$ Electronic supplementary information (ESI) available: Detailed materials and methods, additional spectra of the butterfly and the replica, SEM, TEM and SAXS and left/right hand circularly polarized light (figure and animation). See DOI: $10.1039 / \mathrm{clcc} 11637 \mathrm{a}$ include the exoskeleton of beetles, ${ }^{12}$ the chitin wing scales of butterflies $^{13}$ and in the feathers of birds. ${ }^{14}$

Amongst these are the 3-D structured wing scales of butterflies belonging Callophrys genus, including C. rubi. Studies using SEM, TEM, small-angle XRD and electron tomography ${ }^{15-17}$ confirm that their chitin wing-scales contain polycrystalline domains, each a 3-D bicontinuous cubic photonic crystal. Within each domain, chitin and air form mutually interpenetrating sub-volumes, with their interface sharing the topology and symmetry of the gyroid minimal surface with space group Ia $\overline{3} \mathrm{~d}$. The space group of both sub-volumes, as opposed to that of the dividing surface, is $\mathrm{I} 4{ }_{1} 32$, a non-chiral sub-group of Ia $\overline{3} \mathrm{~d}$. While the space group for each mutually interpenetrating sub-volume is the same, their respective screw axes are of opposite hands. The chiral substructure is most easily seen when the architecture of one sub-volume is represented as a net with three-fold connectivity, known as the srs net after the $\mathrm{SrSi}_{2}$ structure (Fig. S1 $\dagger$ ). ${ }^{18}$ This network contains two chiral sub-networks along the 100 and 111 crystallographic directions respectively, each with opposite chirality. Saba et al. numerically solved Maxwell's equations to extract reflection spectra as a function of solid volume fraction at fixed dielectric contrast (2.4) and unit cell size $(310 \mathrm{~nm})$ for such structures. They specifically confirm by theory that gyroid photonic crystals display circular dichroism. ${ }^{17}$

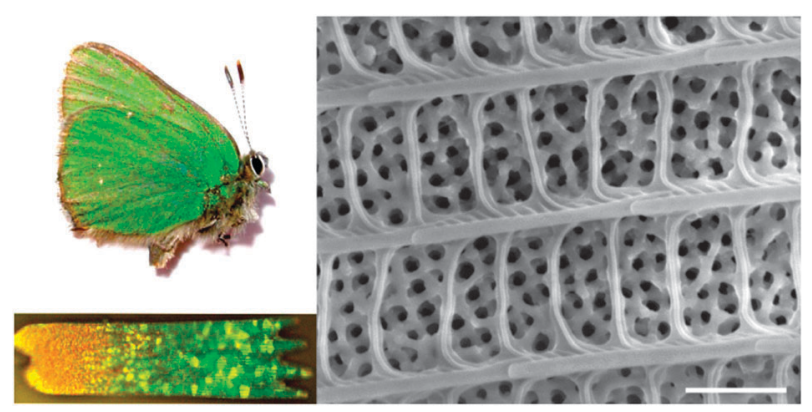

Fig. 1 (a) C. rubi with its green wings. (b) Light micrograph of a single wing scale. In the distal part of the scale (right hand side of the image) mosaic green domains but also yellow and blue can be observed. They originate from the $3 \mathrm{D}$ gyroid structures found below the longitudinal ridges. The size of a wing scale is $\sim 50 \times 200 \mu \mathrm{m}$. (c) SEM micrograph of the top of the wing scale. The ridges and cross ribs are seen as well as an underlying porous structure. Scale bar is $1 \mu \mathrm{m}$. 


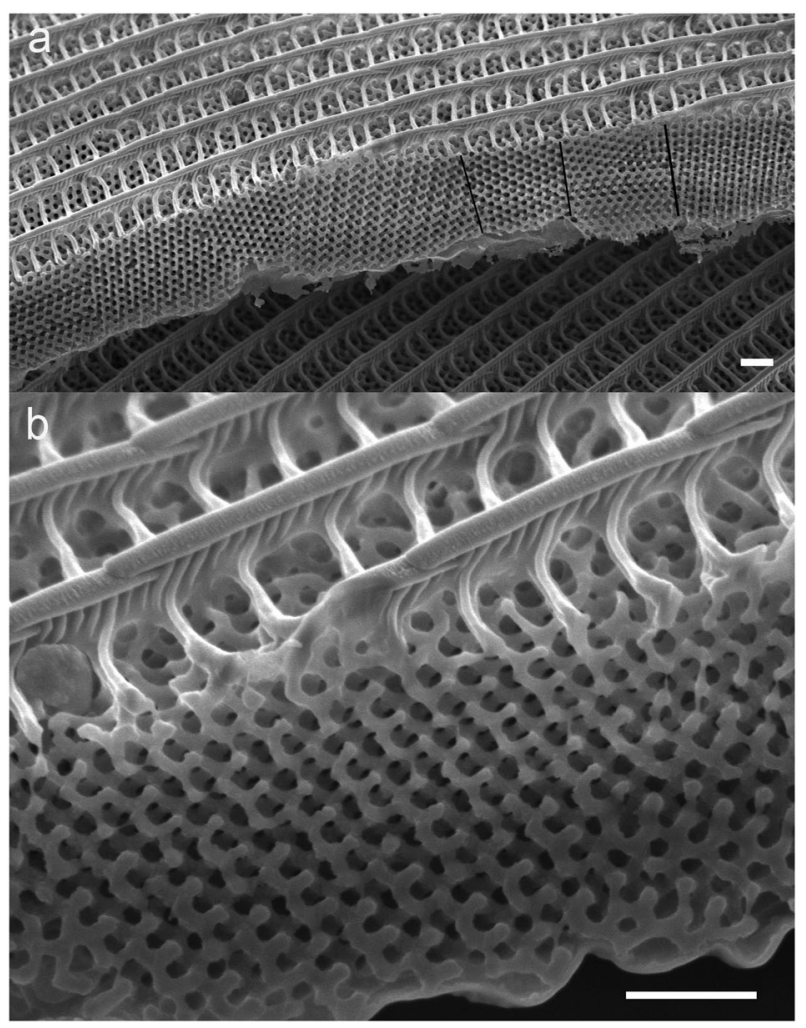

Fig. 2 SEM micrograph on a cross sectional view of a butterfly wing scale. A highly ordered, porous, three-dimensional structure extends throughout the scale, beneath the ribs and the cross-ribs. At least 7 distinct domains of $\sim 2-3 \mu \mathrm{m}$ in length can be seen in the cross section. Each domain is associated with a colour in the mosaic structure seen in Fig. 1b. Black lines have been added for clarity. (b) The same structure at higher magnification. Scale bar is $1 \mu \mathrm{m}$.

Here we use the chiral structures of the wing scale of $C$. rubi as template to produce chiral silica replicas with the gyroid structure. We also characterise the morphology and photonic response of these biomimetic, inorganic materials. A sol-gel method similar to Bartl et al. ${ }^{19}$ were used to synthesize the replica. Samples were cross-section polished and characterized using FESEM. The spectral response was measured with a microreflectometer. The macroscopic colour of C. rubi wings is independent of the angle of incident light and thus appears green from all directions (Fig. 1a). Upon magnifying the wing-scale, a mosaic-like pattern of distinct green (dominant), yellow and blue colours can be seen. Images obtained with SEM (Fig. 1c) from the dorsal side of the wing scales shows a cubic bicontinuous structure beneath the longitudinal ridges and cross ribs. When the butterfly is cut perpendicular to the wing, crystalline domains are visible (Fig. 2) with a calculated unit cell of $339 \pm 5 \mathrm{~nm}$ (Fig. S4†).

The domains, outlined in $2 \mathrm{a}$, are in the range of $2-3 \mu \mathrm{m}$ and responsible for the distinct mosaic-colours seen in Fig. $1 b$. Reflectance spectra collected at different positions along the scale with the field diaphragm fully closed also confirms this observation (Fig. S2†). All 3-D photonic crystallite domains in these wing scales have the gyroid structure in different orientations. Each differently oriented domain has a unique reflectance spectrum, in the range from violet to yellow-green $(400-570 \mathrm{~nm})$ which, on averaging over multiple domains, gives rise to the characteristic green colour of the butterfly. ${ }^{20}$

In the templating process, the sol gel silica fills up the structure and, by using a sandwich construction, all excess silica is transported away from the wing through capillary action. After removal of the organic material by acid etching an inverse structure is obtained, with air in place of the chitin and silica where it was previously air. An apparent increase in the fill fraction was observed after replication. This is expected given the previous reports for the fill fractions of C. rubi, i.e. $0.2-0.4 .^{15,21}$

During the templating process the ordered interior, responsible for the colour, are replicated to a high fidelity with the domains visible in both optical and SEM micrographs (Fig. 3). During the replication process only the gyroidal regions within the wing scale were templated, and not the ridges and cross-ribs. The most prominent colours reflected from the distal part of the silica scale are cyan and blue. Calculations show that a blue shift is expected when either the fill factor or the dielectric contrast are decreased., ${ }^{4,22,23}$ The silica has an equivalent unit cell, an increased fill fraction and decreased dielectric constant relative to its chitin template. ${ }^{24}$ Thus the change in the dielectric contrast is the main factor for the observed blue shift upon replication (Fig. 4).

In addition to a partial band gap in the visible spectral region, the replicated wing scales show optical activity, as confirmed by imaging the sample under cross polarized light (Fig. 5). The optically amorphous nature of the silica (Fig. S3†) and the intrinsic cubic symmetry of the structure rules out birefringence as the source of the reflected light. More significantly, circular dichroism was confirmed by observing differences in the handedness of the reflected light (Fig S6 and Anim. S1 $\dagger$ ). This effect arises from the chirality of the screw axes in the srs-net, which preferentially reflects left or right circularly polarized light depending on the

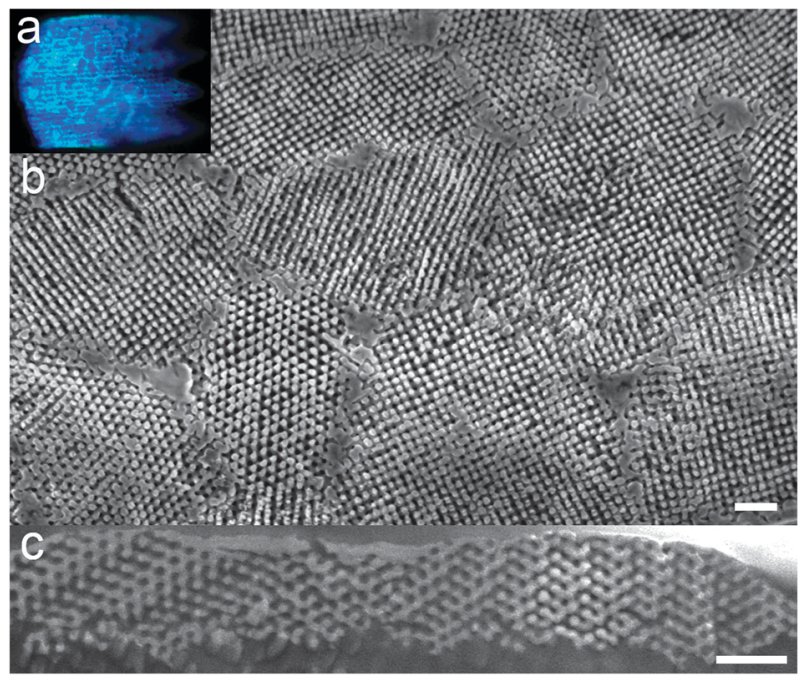

Fig. 3 Silica replicas (a) Light micrograph showing blue and cyan domains. Only the distal part is replicated. The size is $\sim 50 \times 100 \mu \mathrm{m}$. (b) SEM image showing the ventral view with highly ordered regions in a mosaic like pattern. (c) SEM image showing different domains of a cross sectioned polished replica embedded in epoxy resin. Scale bar is $1 \mu \mathrm{m}$. Unit cell is $330 \pm 15 \mathrm{~nm}$. 


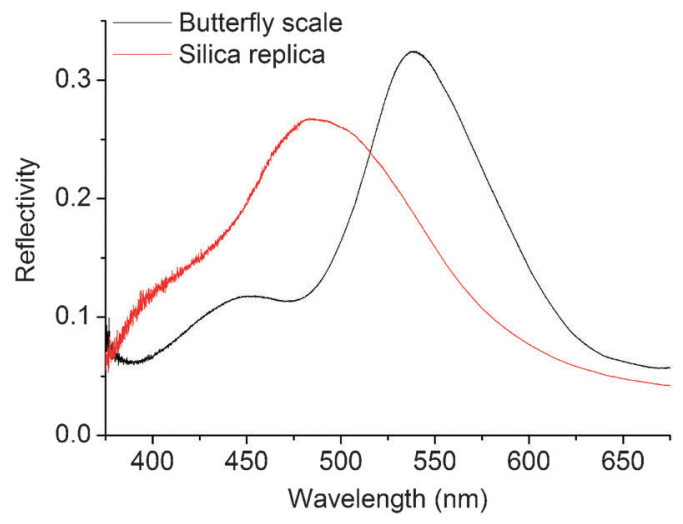

Fig. 4 Representative reflectivity spectra of a single C. rubi wing scale and its silica replica. Note the distinct peak at $\sim 540 \mathrm{~nm}$ in the butterfly scale and the blue-shift to $\sim 480 \mathrm{~nm}$ observed in the replica.

crystallographic direction of the lattice, e.g. the 100 or the 111 direction.

The photonic response in both the replica and its template demonstrates that neither display full photonic band gaps (i.e. $100 \%$ reflectivity). Band gap calculations suggest a dielectric constant of at least 6 is required to achieve a full photonic band gap the is independent of the angle of incidence, given a unit cell and fill fraction similar to that of C. rubi. ${ }^{4,22}$

Other materials with dielectric contrasts greater than 6 have been used in replication of butterfly wing scales, e.g., titania. ${ }^{25}$ However, the formation of crystals during the sintering process could introduce defects that render the replica ineffective for optical applications. A potential route to synthesize defect free high refractive index templates is to infiltrate the wing scales with monodisperse nanoparticles. Super-paramagnetic nanoparticles for example would potentially lead to a structure with not only full band gaps but also magneto-optic effects. ${ }^{26}$ Finally, these chiral structures may have interesting applications for metamaterial design, where a number of extraordinary effects have been predicted. ${ }^{27}$

A 3-D, chiral photonic crystal of silica has been synthesized using the butterfly $C$. rubi as a template. To our knowledge,

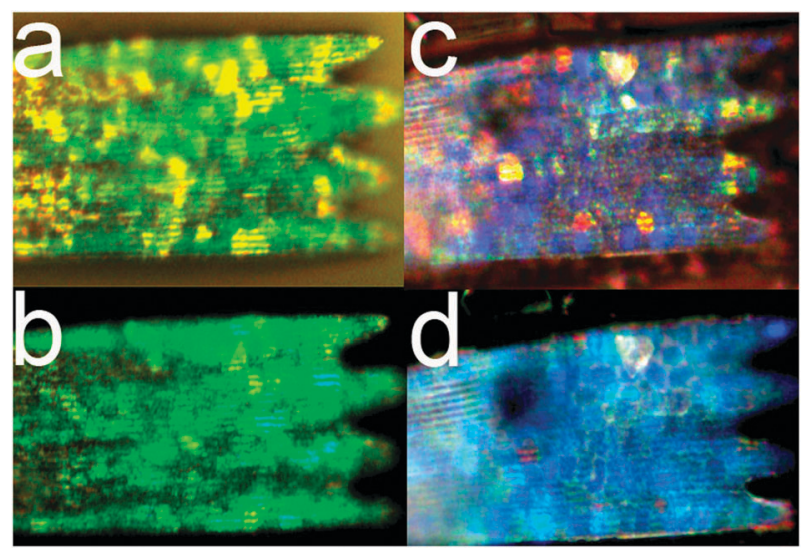

Fig. 5 (a) Butterfly wing scale under non-polarized light and (b) under cross-polarized light (c) A silica replica under non-polarized light and (d) under cross-polarized light. Both show optical activity, supporting its chirality (see Fig. S6†). this is the first inorganic gyroid structure with a unit cell size of photonic length scale. The structure and long range order of these photonic crystals was confirmed by SEM and reflectivity, and these were also shown to be optically active.

We thank Dr J. Bergsten for the butterfly specimens and Dr T. Plivelic for assistance with the I711 beamline at MAXlab. $\mathrm{CM}$ and RWC acknowledge financial support from the EXSELENT Centre, Stockholm University. ECT acknowledges financial support from the Swedish Research Council (VR), and the EU Marie Curie Reintegration grant FP7- PEOPLEERG-2008. The Knut and Alice Wallenberg foundation are acknowledged for supporting the electron microscopy facilities at SU.

\section{Notes and references}

1 E. Yablonovitch, J. Opt. Soc. Am. B, 1993, 10, 283-295.

2 T. F. Krauss and R. M. De La Rue, Prog. Quantum Electron., 1999, 23, 51-96.

3 A. Schoen, Technical Report, NASA, Washington, DC, 1970.

4 L. Martin-Moreno, F. J. Garcia-Vidal and A. M. Somoza, Phys. Rev. Lett., 1999, 83, 73-75.

5 J. H. Moon and S. Yang, Chem. Rev., 2009, 110, 547-574.

6 K. Larsson, K. Fontell and N. Krog, Chem. Phys. Lipids, 1980, 27, 321-328.

7 S. Hyde, S. Andersson, K. Larsson, Z. Blum, T. Landh, S. Lidin and B. W. Ninham, Chemistry and Biology, Elsevier, Amsterdam, 1997.

8 B. E. S. Gunning, Protoplasma, 2001, 215, 4-15.

9 Y. Deng and M. Mieczkowski, Protoplasma, 1998, 203, 16-25.

10 E. L. Snapp, R. S. Hegde, M. Francolini, F. Lombardo, S. Colombo, E. Pedrazzini and N. Borgese, J. Cell Biol., 2003, 163, 257-269.

11 P. Vukusic and J. R. Sambles, Nature, 2003, 424, 852-855.

12 J. W. Galusha, L. R. Richey, J. S. Gardner, J. N. Cha and M. H. Bartl, Phys. Rev. E: Stat., Nonlinear, Soft Matter Phys., 2008, 77, 50904.

13 H. Ghiradella, Appl. Opt., 1991, 30, 3492-3500.

14 M. D. Shawkey, V. Saranathan, H. Pálsdóttir, J. Crum, M. H. Ellisman, M. Auer and R. O. Prum, J. R. Soc. Interface, 2009, 6, S213.

15 K. Michielsen and D. G. Stavenga, J. R. Soc. Interface, 2008, 5, 85.

16 V. Saranathan, C. O. Osuji, S. G. J. Mochrie, H. Noh, S. Narayanan, A. Sandy, E. R. Dufresne and R. O. Prum, Proc. Natl. Acad. Sci. U. S. A., 2010, 107, 11676.

17 M. Saba, M. Thiel, M. D. Turner, S. T. Hyde, M. Gu, K. GrosseBrauckmann, D. N. Neshev, K. Mecke and G. E. Schröder-Turk, Phys. Rev. Lett., 2011, 106, 103902.

18 D. Friedrichs, M. O'Keeffe and O. M. Yaghi, Acta Crystallogr., Sect. A: Found. Crystallogr., 2002, 59, 22-27.

19 J. W. Galusha, L. R. Richey, M. R. Jorgensen, J. S. Gardner and M. H. Bartl, J. Mater. Chem., 2010, 20, 1277-1284.

20 K. Michielsen, H. De Raedt and D. G. Stavenga, J. R. Soc. Interface, 2009, 7, 765.

21 G. E. Schroder-Turk, S. Wickham, H. Averdunk, F. Brink, J. D. Gerald, L. Poladian, M. C. J. Large and S. T. Hyde, J. Struct. Biol., 2011, 174, 290.

22 K. Michielsen and J. S. Kole, Phys. Rev. B: Condens. Matter, 2003, 68, 115107.

23 M. Maldovan, A. M. Urbas, N. Yufa, W. C. Carter and E. L. Thomas, Phys. Rev. B: Condens. Matter, 2002, 65, 165123.

24 The refractive index of the mesoporous silica (1.23 \pm 0.05 see Fig. $\mathrm{S} 3 \dagger)$ is lower than chitin $(1.55+i 0.0622)$.

25 M. R. Weatherspoon, Y. Cai, M. Crne, M. Srinivasarao and K. H. Sandhage, Angew. Chem., Int. Ed., 2008, 47, 7921-7923.

26 J. B. González Díaz, A. García Martín, J. M. García Martín, A. Cebollada, G. Armelles, B. Sepúlveda, Y. Alaverdyan and M. Käll, Small, 2008, 4, 202-205.

27 J. Zhou, J. Dong, T. Koschny, M. Kafesaki and C. M. Soukoulis, Phys. Rev. B: Condens. Matter Mater. Phys., 2009, 79, 121104. 